\title{
231988
}

UCRLJC-128424

PREPRINT

\section{Scrape-off Layer Turbulence Theory and Simulations}

\author{
$\mathbf{X . Q .} \mathbf{X u}$ \\ R.H. Cohen
}

This paper was prepared for submittal to

Plasma Edge Theory Conference

Oxford, England

September 15-17, 1997

September 4, 1997

This is a preptint of a paperintended for publlontion in a joumal or proceedings. Since changes may be made before publication, this preprint is made avallable with the understanding that it will not be cited or reproduced without the permission of the author. 


\section{DISCLAMMER}

This document was prepared as an account of work sponsored by an agency of the United States Government. Neither the United States Government nor the University of California nor any of their employees, makes any warranty, express or implied, or assumes any legal liablitity or responsiblity for the accuracy, completeness, or usefulness of any information, apparatus, product, or process disclosed, or represents that its use would not infringe privately owned rights. Reference herein to any specific commercial product, process, or service by trade name, trademark, manufacturer, or otherwise, does not necessarily constitute or imply its endorsement, recommendation, or favoring by the United States Government or the University of California. The views and opinions of authors expressed herein do not necessarily state or reflect those of the United States Government or the University of California, and shall not be used for advertising or product endorsement purposes. 


\title{
Scrape-Off Layer Turbulence Theory and Simulations
}

\author{
X.Q. Xu and R.H. Cohen
}

Lawrence Livermore National Laboratory, University of California, Livermore, CA 94550 USA

\begin{abstract}
Significant investigations in the area of scrape-off layer (SOL) turbulence theory and simulations are reviewed. The review begins with description and derivation of the various models investigating specific linear modes as well as a discussion of the region of validity. Special attention is focused on various low-frequency electromàgnetic drift-types modes in an x-point divertor geometry, which are generally believed to be relevant under normal operating conditions for current and future large fusion devices. Benchmarks of linear ballooning codes with nonlinear 3D fluid code will be given. The anomalous transport from simulations and mixing length estimates are discussed, and a summary of relevant experimental results as well as imperical values in the transport codes are given. Studies of mechanisms for the $\mathrm{L}-\mathrm{H}$ transition due to turbulent transport in the SOL and its impact on the the H-mode power threshold are also surveyed.
\end{abstract}

\section{Introduction}

Scrape-off-layer (SOL) turbulence is observed in most fusion discharges and produce anomalous radial transport of particles and energy from the edge region of the confined plasma. Its obvious signature is large amplitude fluctuations of plasma density, temperature, and plasma potential. The study of these fluctuations is important, as they play a central role in determining the SOL width, maybe also strongly impact the detachment of divertor plasma, $\mathrm{L}-\mathrm{H}$ transitions, and edge localized mode (ELMs). In this review we shall survey recent theoretical developments for SOL turbulence, with particular emphasis on progress made since Ref.[6].

The organization of this review is as follows. In Sec. 2 we give a set of basic equations for the most important classes of instabilities in SOL. The major instability mechanisms will be surveyed. We also briefly review instabilities which do not fit into this framework. Particular emphasis will be given to the effects resulting from inclusion of the electromagnetic perturbation. In Sec. 3, we summarize work on L$\mathrm{H}$ transitions based on SOL turbulence. In Sec. 4, we discuss $\mathrm{X}$ point physics and $3 \mathrm{D}$ simulation results with a realistic divertor geometry. An overall summary and discussion is in Sec.5.

\section{Physics Models and Dynamic equations}

In low- $\beta$ plasmas, there are many sources of turbulence, such as gradients of density, temperature, parallel ion velocity, radiation and ionization. The most studied instabilities for the SOL are sheath-driven conducting-wall modes and interchange modes. It wsa noted in [6] that these are not really independent modes, but rather 
separat two co-existing mechanisms for destabilizing drift-wave type instabilities. In fact, since typical SOL plasmas have low densities, and temperatures, and steep radial gradients, the diamagnetic drift time scale, collision time scale, Alfven time scale, and electron transit time scale are all of the same order, there is no clear distinction among ideal MHD, resistive MHD and Alfven modes. They are all drift-wave type instabilities with full electromagnetic perturbations. The table I gives most important spatial scale lengths and times for the typical SOL plasmas. Thus an approximated set of equations to describe the turbulence is given by a normalized five-field model obtained reduction of the Braginskii equations:

$$
\begin{aligned}
& \frac{\partial}{\partial t} \hat{\varpi}+\nabla \cdot\left[\left(\hat{\mathbf{U}}+\hat{\mathbf{U}}_{P_{i}}+\hat{V}_{i}\right) \hat{\boldsymbol{\varpi}}\right]=2(1+\tau) \frac{\mathbf{b} \times \hat{\kappa} \cdot \hat{\nabla} \hat{P}_{e}}{\hat{n}}+\hat{\nabla}_{\| \hat{j}_{\|}}+\hat{\mu}_{i i} \hat{\nabla}_{\perp}^{2} \hat{\varpi} \\
& \frac{\partial}{\partial t} \hat{n}+\nabla \cdot\left(\left(\hat{\mathrm{U}}+\hat{V}_{i}\right)\left(\hat{n}+\hat{N}_{0}\right)\right)=2 \frac{\rho_{s}}{R_{0}} \mathrm{~b}_{0} \times \hat{\kappa} \cdot \hat{\nabla} \hat{P}_{e}+\frac{\rho_{s}}{R_{0}} \hat{\nabla}_{\| \hat{j}_{\|}}-\frac{\rho_{s}}{R_{0}} \hat{\nabla}_{\|}\left(\hat{n} \hat{V}_{i}\right)(2) \\
& \frac{\partial}{\partial t} \hat{V}_{i}+\nabla \cdot\left(\left(\hat{\mathbf{U}}+\hat{V}_{i}\right)\left(\hat{V}_{i}+\hat{V}_{i 0}\right)\right)=-\frac{\tau \rho_{s}}{\hat{n} R_{0}} \hat{\nabla}_{\|} \hat{n}-\mu_{e} \tau \hat{\nabla} \hat{A} \times \hat{b}_{0} \cdot \hat{\nabla} \hat{N}_{0}+\hat{\mu}_{\|} \hat{\nabla}_{\|}^{2} \hat{V}(3) \\
& \frac{\partial}{\partial t} \hat{T}_{e}+\nabla \cdot\left(\hat{\mathbf{U}}\left(\hat{T}_{e}+\hat{T}_{\mathrm{e} 0}\right)\right)=\chi_{\|}^{c} \hat{\nabla}_{\|}\left(\hat{T}_{e}^{5 / 2} \hat{\nabla}_{\|} \dot{\hat{T}}_{e}\right) \\
& \frac{\partial}{\partial t} \hat{A} j_{p a r a}+\nabla \cdot\left(\hat{\mathbf{U}}\left(\hat{j}_{\|}+\hat{J}_{\| 0}\right)\right)=\left(\hat{T}_{e} \hat{\nabla}_{\| \hat{n}}+1.71 \hat{n} \hat{\nabla}_{\|} \hat{T}_{e}-\hat{n} \hat{\nabla}_{\|} \hat{\phi}\right) / \hat{\beta}_{A}-\hat{\nu}_{e} \hat{j}_{\|} \\
& -\hat{n} \hat{\nabla} \hat{A} \times b_{0} \cdot \hat{\nabla} \hat{\Phi}_{0}+\hat{T}_{e} \hat{\nabla} \hat{A} \times b_{0} \cdot \hat{\nabla} \hat{N}_{0} \\
& +1.71 \hat{n} \hat{\nabla} \hat{A} \times b_{0} \cdot \hat{\nabla} \hat{T}_{e 0} \\
& \hat{\nabla}_{\perp}^{2} \hat{\phi}=\frac{\rho_{s}}{R_{0}} \hat{\omega} \\
& \hat{\nabla}_{\perp}^{2} \hat{A}-\hat{\beta} \hat{A}=-\hat{\beta} \hat{A} j_{\text {para }}, \\
& \hat{j}_{\|}=\hat{A} j_{\text {para }}-\hat{n} \hat{A}, \\
& \hat{\nabla}_{\|}=\hat{\nabla}_{\|}^{0}+\hat{\beta}_{A} \hat{\nabla} \hat{A} \times \mathbf{b}_{0} \cdot \hat{\nabla}, \\
& \hat{\nabla}_{\|}^{0}=R_{0} \mathrm{~b}_{0} \cdot \nabla \text {, } \\
& \hat{\kappa}=R_{0} \mathbf{b}_{0} \cdot \nabla \mathbf{b}_{0} \text {. }
\end{aligned}
$$

where $\tau=T_{i} / T_{e}, \hat{\beta}=\beta \mu_{e} / 2, \mu_{e}=m_{e} / M_{i}, \beta=8 \pi n T_{e} / B^{2}, \chi_{\|}^{c}=4.27 \omega_{b e x}^{2} / \omega_{c i} \nu_{e i}, \omega_{b e x}=$ $v_{T e} / R_{0}, \hat{\mu}_{i i}=\tau \nu_{i i} / 4 \omega_{c i}, \hat{\mu}_{\|}=1.28 \omega_{b i x} / \omega_{c i} \nu_{i i}, \omega_{b i x}=v_{T i} / R_{0}, \hat{\nu}_{e}=\nu_{e i} / \omega_{c i}, \hat{\beta}_{A}=R_{0} m_{e} / \rho_{s} M_{i}, \hat{P}_{e}=$ $\hat{n} \hat{T}_{e}, \hat{\mathbf{U}}=\mathbf{b} \times \nabla \hat{\phi}$, and $\hat{\mathbf{U}}_{P_{i}}=\mathbf{b} \times \nabla \hat{P}_{i} / \hat{n} . \hat{n}=n(r, \theta, \zeta) / n\left(r_{s}, \theta_{0}\right), \hat{\phi}=e \phi / T_{e}\left(r_{s}, \theta_{0}\right), \hat{T}_{e}=$ $T(r, \theta, \zeta) / T_{e}\left(r_{s}, \theta_{0}\right), \hat{V}_{i \|}=V_{i \|}(r, \theta, \zeta) / c_{s}\left(r_{s}, \theta_{0}\right), \hat{j}_{\|}=j_{\|}(r, \theta, \zeta) / n_{e} e c_{s}\left(r_{s}, \theta_{0}\right)$, and $\hat{A}_{\|}=$ $\left(M_{i} / m_{e}\right)\left(A_{\|} / \rho_{s} B_{0}\left(r_{s}, \theta_{0}\right)\right)$.

Note that when $k_{\perp}^{2} \rho_{s}^{2} / \hat{\beta}>>1$ in Eq. (7), the magnetic flutter $\hat{A}$ is small and the fluctuations are electrostatic. Thus the single parameter $\hat{\beta}$ can controls the transition from electromagnetic to electrostatic limit.

\subsection{SOL sheath boundary conditions}

The turbulent sheath fluxes are

$$
\tilde{V}_{i}=\tilde{c}_{s}
$$




$$
\begin{aligned}
& \left.\left.\left.\left.\tilde{j}_{\|}^{e l}\right|_{L}=n_{s} e c_{s}\left\{\frac{U_{0}}{c_{s}} \hat{n}+\left(1-\frac{U_{0}}{c_{s}}\right) \hat{\phi}+\left[\frac{U_{0}}{2 c_{s}}-\left(1-\frac{U_{0}}{c_{s}}\right) \ln \left(\frac{v_{T_{e}}}{2 c_{s} \sqrt{\pi}} /\left(1-U_{0} / c_{s}\right)\right) \hat{T}_{c}\right]\right\}\right),\right\}_{j}\right) \\
& \left.\tilde{j}_{\|}^{e l}\right|_{0}=n_{s} e c_{s}\left\{\frac{U_{0}}{c_{s}} \hat{n}-\left(1+\frac{U_{0}}{c_{s}}\right) \hat{\phi}+\left[\frac{U_{0}}{2 c_{s}}+\left(1+\frac{U_{0}}{c_{s}}\right) \ln \left(\frac{v_{T_{e}}}{2 c_{s} \sqrt{\pi}} /\left(1+U_{0} / c_{s}\right)\right) \hat{T}_{e}\right]\right\},
\end{aligned}
$$

where the current velocity $U_{0}=J_{\|_{0}} /$ ne and $J_{\| 0}$ is the equilibrium SOL current. As noted in [RC], if $\hat{\nu}_{\|}$is strictly zero, the boundary condition on $\hat{V}$ I srequired, as $\hat{V}_{i}->c_{s}$ emerges naturally from gas dynamics considerations. For simulation which inevitably have finite but small $\hat{\eta}_{\|}$, imposing this boundary condition implies that the natural gas dynamic condition is approximated.

Since $\Phi_{0}(x)=\Lambda T_{e 0}(x)$, thus we have the flow velocity

$$
\begin{aligned}
\hat{U}_{0}(x) & =\frac{\partial \hat{\Phi}_{0}}{\partial x}=\Lambda \frac{\partial \hat{T}_{e 0}}{\partial x} \\
\Lambda & =\ln \left(\frac{v_{T_{e}}}{2 \sqrt{\pi} c_{s}} \frac{1}{1+\frac{U_{0}}{c_{s}}}\right)+\text { higherorder. }
\end{aligned}
$$

This analysis ignores the effect of the small angle incidence of magnetic field lines on the walls [Cohen and Dimitri]. When this effect is included, perturbed $\mathbf{E} \times \mathbf{B}$ and dymagnetic currents enter into the boundary condition [CR]. This affects growth rates and mode structure; nonlinear consequences have not yet been studied.

\subsection{Critical Parameters to Control Turbulence}

With the neglect of atomic process and Debye-scale phenomena, SOL turbulence is controlled by dimensionless parameters. $\hat{\beta}$ is a key parameter to control the transition between the electromagnetic and electrostatic limits. $\mathbf{U}_{\mathbf{p i}}$ is the lowest order ion gyroradius effect which yields the finite ion diamagnetic drift stabilization. $\left(L_{n}^{-1}=-(1 / n)\left(\partial N_{0} / \partial r\right), L_{\overline{\bar{T}}}-\left(1 / T_{e}\right)\left(\partial T_{e 0} / \partial r\right)\right) / \rho_{s}$ parameterize the linear instability drivers. $L_{T e} / \rho_{s}$ is also associated with velocity shear $\partial V_{E 0} / \partial r . \rho_{s} / R_{0}$ gives the magnitude of curvature. Defining $L_{\|}=q R_{0}, L_{\|} / \rho_{s}$ is the connection length. $\hat{s}$ is shear stabilization (fanning effects) in the SOL. $\hat{\nu}_{e}$ is a key parameter to describe the electron parallel motion. For fixed $\hat{\beta}, \hat{\nu}_{e}$ determines whether the turbulence is in the electromagnetic or electrostatic limit, depending on the ratio $\nu_{e i} / \omega$. The high parallel electron heat conduction can smooth perturbations of $\hat{T}_{e}$ along the magnetic field lines in the electrostatic limit. $\Lambda$ is the sheath boundary parameter, which characterizes the effectiveness of sheath impedance as well as biasing.

From these combinations of the parameters, one can expect the following general expression for the turbulent transport coefficients:

$$
\chi_{\perp}=\chi_{G B} F\left(\hat{\beta}, \rho_{s} / R_{0}, L_{\perp} / \rho_{s}, L_{\|} / r h o_{s}, \hat{\nu}, \Lambda\right)
$$

Here $\chi_{G B}=\left(c T_{e} / e B_{0}\right)\left(L_{\perp} / \rho_{s}\right)$ is the gyro-Bohm coefficient, $L_{\perp}$ is density, temperature, parallel velocity or ion pressure gradient scaling length $L_{n}, L_{T e}, L_{v}$, or $L_{P i}$. 
$\mathrm{F}$ is a dimensionless function of the dimensionless arguments to be determined from theoretical arguments and/or from serial runs of simulations.

\subsection{Linear modes}

For typical SOL plasmas, the steep radial gradients of density and temperature are the source of instabilities. Fluctuations of the plasma pressure generate fluctuating perpendicular currents according to $\nabla P=(1 / c) j_{\perp} \times \mathbf{B}$. Owing to the charge neutrality condition, $\nabla \cdot \mathrm{j}=0, \mathrm{j}_{\perp}$ causes fluctuating parallel currents $\mathrm{j}_{\|}$in toroidal plasmas. The parallel current is compensated by non-divergence-free perpendicular polarization currents. The drift -wave instabilities are developed . In the SOL, the sheath boundary condition yields a constraint for the parallel current $j_{\|}$. In the electromagnetic regime, the parallel current $j_{\|}$introduces small-scale perturbations of the magnetic field, $\nabla \times \mathbf{B}=(4 \pi / c) j_{\|}$, which enable electrons to move perpendicular to the equilibrium magnetic field lines. However, the perturbed magnetic field in turn produces a counter-current to impede the fast changes in $\mathbf{j}_{\|}$. through $\nabla \times \mathbf{E}_{\|}=-(1 / c) \partial \mathbf{B}_{\perp} / \partial t$. This is the essence of the skin effect. Taking into account of electron conductivity and inertia as given by the full electron momentum equation in Eq. (1)-(10), the perturbed magnetic field is described by

$$
\frac{\partial \mathbf{B}}{\partial t}=\left(1-i \frac{\omega}{\nu_{e i}}\right) \frac{c^{2}}{4 \pi \sigma} \nabla_{1}^{2} \mathbf{B}+\nabla \times(\mathbf{V} \times \mathbf{B}) .
$$

The first term is the electron skin effect. If $\omega \ll \nu_{e i}$, the perturbed magnetic energy dissipates into plasma. One other hand, if $\omega \gg \nu_{e i}$, the total magnetic energy is increased, which is a stabilizing effect. This collisonless stabilizing effect is equivalent to a finite ion gyroradius effect. The fluctuations over the collisionless depth $\delta=c / \omega_{p e}$ is smoothed by perpendicular electron motion due to the perturbed magnetic field. The characteristic spatial scale lengths and time are given in table. 1.

Our reduced equation set includes drives for (1) Conducting-wall modes, (2) KelvinHolmholtz modes, (3) Curvature-driven ideal MHD ballooning and resistive ballooning modes, (4) resistive drift modes, (5) $\nabla_{x} v_{\|}$modes, and, (6) axial shear modes $\left(\nabla_{\|} \mathbf{V}_{\mathbf{E} \times \mathbf{B}}\right)$. These modes can be either electromagnetic or electrostatic depending on the value of the plasma $\beta$. There are two ways in which $\beta$ comes into play: through (a) the skin effect (either collisional or collisionless), (b) MHD instabilities caused by the pressure gradient.

In order to access the importance of finite $\beta$ effects, we now consider the parallel componeut of the electron momentum balance equation ( the generalized parallel Ohm's law) in Eq. (5). Linearizing Eq. (8), using $\tilde{B}=\nabla \times \tilde{A}_{\| !} \mathbf{b}, \tilde{J}=-\frac{c}{4 \pi} \nabla_{\perp}^{2} \tilde{A}_{\|}$, and considering perturbations of the type $\exp [i(\mathbf{k} \cdot \mathbf{r}-\omega t)]$, we obtain the perturbed parallel current

$$
\hat{j}_{\|} \simeq i \frac{k_{\perp}^{2}}{\hat{\beta}} \frac{1}{\Omega \hat{\beta}_{A}} \frac{\hat{T}_{e} \hat{\nabla}_{\|} \hat{n}+1.71 \hat{n} \hat{\nabla}_{\|} \hat{T}_{e}-\hat{n} \hat{\nabla}_{\|} \hat{\phi}}{\left(1-\frac{\omega_{e}^{*}}{\Omega}-\alpha_{T} \frac{\omega_{T_{e}}^{*}}{\Omega}\right)+\left(\frac{\hat{k}_{t}^{2}}{\hat{\beta}^{2}}\right)\left(1+i \frac{\nu_{e i}}{\Omega}\right)}
$$


This equation is rich in physics. It is not just $\beta$ alone that determines whether SOL plasma is electromagnetic or electrostatic. It is the combination of $1 / \beta^{*}=\left(\frac{\hat{k}_{1}^{2}}{\hat{\beta}}\right)(1+$ $\left.i \frac{\nu_{\text {ei }}}{\Omega}\right)$. If $\beta^{*} \ll(\gg) 1$, we obtain the electrostatic (electromagnetic) limit. We would like to point out that even in the intermediate- $\beta$ plasmas where $\beta \sim 1$, we may still in the electrostatic regime at very low temperature where $\nu_{e i} \gg \Omega$. For typical SOL plasma and turbulence parameters, $B=2.0 \mathrm{~T}, n_{\mathrm{e} 0}=3 \times 10^{19} / \mathrm{m}^{3}, T_{\mathrm{e}}=100 \mathrm{eV}, \hat{k}_{\perp} \sim 0.1$, $\beta \sim 3 \times 10^{-3}$, and $\hat{\beta} \simeq 0.6$. Therefore $1 / \beta^{*}=1.7 \times 10^{-4} \ll 1$, the fluctuations are electromagnetic. The parameter $\beta^{*}$ represents the electron skin effects. It is the ratio of skin depth $\Delta_{\text {skin }}$ to turbulence wavelength $\lambda$. For given plasma parameters, the ideal ballooning modes have long wave length $k_{\perp}^{2} \rho_{s}^{2} / \hat{\beta}\left(1+i \nu_{e i} / \Omega\right)<1$ while drift-wave type instabilities have short wave length $k_{\perp} \rho_{s} / \hat{\beta}\left(1+i \nu_{e i} / \Omega\right) \sim 1$.

The simple physical argument in Ref[4] would suggest that the skin effects suppress turbulence. The skin effect is associated with an impedance to the flow of current along the field line which is necessary to compensate charges pumped into the flux tube due to perpendicular non-àmbipolar mechanisms. A linear analysis of electromagnetic modes in the SOL driven by the combined effects of unfavorable curvature and pressure gradients was performed by Kerner, Nassigh and Pogutse. They show that instabilities with a growth rate of the same order as ideal modes can be developed with little field-line bending, due to the presence of a plasma-wall interface. In recent three-dimensional electromagnetic computations of turbulence by Scott et al and by Rogers et al it was found that the turbulence itself actually becomes stronger with increasing $\beta$. We have found that in the flute limit of the conducting wall mode, the electromagnetic and electrostatic regime yield identical results because the electron momentum equation drops out the problem [BCRTX].

It is commonly supposed that plasma instabilities are electrostatic in low beta plasma and electromagnetic in finite beta plasmas. Ideal MHD ballooning modes then exist only within a certain ranges of plasma beta values. Including more complete electron physics as Eq.(5), Rogers et al and Kerner et al have recently shown that drift resistive instabilities exist for any values of $\beta$. But the experiments indicate that there is the critical $\beta$, such as ELMs. An understanding of this paradox is needed. Our understanding is the following. Even though there is no hard $\beta$ threshold limit for instabilities, the growth rate increase rapidly as $\beta$ approaches the ideal ballooning threshold for long wavelength modes.

\subsection{D turbulence simulations}

We have developed a 3D fluid simulation code TEDGE of tokamak edge turbulence with realistic parameters in a DIII-D divertor geometry. The basic set of equations is Eq. (1)-(10) with the sheath boundary conditions Eq.(11). We employ DIIID divertor geometry by reading geometry data from UEDGE code. In order to simulate the high-n drift wave modes, The simulation is performed in aflux ribbon that spans the separatrix. One of the coordinates, $x$, is taken to be orthogonal to the flux surface. The second coordinate, $y$ is the poloidal distance along a field line. The third coordinate, $z$, is the toroidal angle. The simulation volume contains SOL, core 
and private flux regions. The slab geometry in the 2-D poloidal plane is similar to that of UEDGE code. The boundary conditions are bounded in $x$, sheath boundaries in $y$, and periodic in $z$. The code solves all the equations simultaneously with a fully implicit Newton Krylov iteration scheme except field solvers for potential. Some of the results are also obtained from a limiter.geometry with a constant magnetic shear.

We have conducted series simulations by variations of the control parameters in Sec 2.2. We present a comparison of turbulence saturation levels and transport in table II for different values of $\hat{\beta}$. Electromagnetic turbulence produces the higher fluctuation levels than electrostatic one. The most striking quantity is the electron temperature fluctuation level $\hat{T}^{E M} \gg \hat{T}^{E S}$ because the electron parallel conduction is not included in electromagnetic simulation while it does in the electrostatic. The argument is given in Ref.[BCRTX]. It is also interesting to point out that the parallel perturbed current $\hat{j}_{\|} \gg 1$. It is a indication that the magnetic perturbation is important.

\subsection{SOL Transport Scaling}

The goal of SOL turbulence studies is formulation of physics-based transport relations that are capable of reproducing experimental observations and designing new experiments. This task requires consideration of both cross-field and parallel transport mechanisms. As we described in previous sections, the turbulence analysis and modeling would yield the anomalous cross field transport. The magnitude of the transport depends on the density and temperature profiles. However, a direct parameterization of the magnitude of the cross-fields transport in terms of local values of density, temperature, magnetic field, etc. would be most desirable. Assuming parallel transport is classical, the standard approach to determine the scaling of local cross-field transport with local plasma conditions is to solve the equation for the conservation of energy $\nabla_{\|} \cdot q_{\|}+\nabla_{\perp} \cdot q_{\perp}+Q_{R a d}=0$ for the scale length of the cross-field temperature profiles $L_{T e}$. Substituting the scale lengths $L_{T e}$ into the anomalous cross field transports yields the desired scaling. In table III, we list scaling dependence on the local plasma density $n$, temperature $T_{e}$, magnetic field $B$, and connection length $L$ as $\chi_{\perp} \sim n^{\alpha} B^{\beta} L^{\delta} T_{e}^{\sigma}$ for vaious current generation machines for $L$ mode and available model anomalous transport. It shows a consistent trends on inverse dependence on density $n$ and connection length $L$ for different machines, independent of magnetic field $B$ for C-MOD and ASDEX. But there is discrepancy with temperature $T_{e}$ dependence. This discrepancy mainly comes from what parallel heat transport model is used. For DIII-D and flux limited parallel heat flux, $\sigma=-0.25$. While for Spitzer-Haarm parallel heat flux, $\sigma=1.25$. For different model-specific theoretical scalings, there are consistent trends with respect to dependence on magnetic field $B$, temperature $T_{e}$ and density $n$ for collisionless plasmas. However there is either weak or no correlation between theory and experiments.

\section{SOL based L-H transtion models}

Tokamak plasmas exhibit states of low (L) and high (H) confinement. $\mathrm{H}$ mode plasmas have better core confinement, sharper edge electron density profiles, low 
edge fluctuation levels and turbulence fluxes, and large rotational velocity. There is typically a threshold input power required to obtain enhancement $\mathrm{H}$ mode.

\subsection{The mechanism for the L-H transition}

The most theoretical work on $\mathrm{L}-\mathrm{H}$ transition machnisms has focused on effects in the "edge" region inside the separatrix-e. g. rotational shear stabilization of, and generation by, turbulence[Diamond], and generation of local negative magnetic shear by finite- $\beta$ shift of flux surfaces[Drake].

In the past few years, however there have been several models based on turbulence in the SOL, we review these here. L-mode plasmas are characterized by higher-amplitude turbulence than H-mode plasmas. The challenge is to identify the underlying instabilities which are responsible for L-mode transport and the mechanisms which can be used to stabilize the modes in the $\mathrm{L}-\mathrm{H}$ transition. In our early study, we found that increasing the radial mode number has a stabilizing effect on the conducting - wall and curvature driven interchange modes in a tokamak SOL, arising from the increased polarization response. Such an effect is naturely imposed as the SOL width is decreased. For a narrow-enough SOL, the stabilizing effect is stronger than the increase in the instability drives. The well-known criterion for velocity shear stabilization is $\frac{d V_{0}(r)}{d r}>\gamma_{\max }$, where $\gamma_{\max }$ is maximum linear growth of instabilities. Based on flow shear stabilization mechanism and the intrinsic flow shear in SOL, Cordey et al pointed out that the criterion for stability of interchange modes leads to the condition $\hat{\rho}=\rho_{s} / L_{s o l}>C$ where $C$ is of the order of unity. An explanation for the L-H transition through an electromagnetic mechanism (suppression of drift wave turbulence by the skin effect) is offered by Chankin. Since drift turbulence is accompanied by fluctuating parallel currents, the skin effect is capable of reducing its level by splitting wide flux tubes. The criterion for a strong influence of the collisional skin effect on the drift turbulence corresponds to $\omega \simeq \omega_{\text {coll }, \text { skin }}=c^{2} / 8 \sigma \Delta^{2}$ for fluctuations with frequency $\omega$ and a radial displacement $\Delta$. The necessary condition for the suppression of turbulence in other limit is that collisionless skin depth $\Delta_{s k i n} \sim c / \omega_{p e}$ must be smaller than the size of drift fluctuations $\Delta_{\text {drift }}: \Delta_{g k i n}<\Delta_{\text {drift }}$.

\subsection{H-mode power threshold}

The transition from $\mathrm{L}$ to $\mathrm{H}$ mode typically occurs when the auxiliary heating power exceeds a threshold value. Once we obtain transport coeficients from theory, the threshold power should scale as $P_{\text {thres }} \sim R^{2} n D_{1}\left(T_{e}+T_{i}\right) / \Delta_{\text {sol }}$. We can compare such scalings with what obtained from the ITER confinement Database and Modeling expert Group's fit to their multi-machine database

$$
P_{\text {thres }}=0.45 B(T)\left(n_{20}\right)^{0.75}[R(m)]^{2}\left[0.6\left(n_{20}\right) R(m)^{2}\right]^{ \pm 0.25} M W .
$$

But recent JET data show different density and major radius scaling: $P_{\text {thres }} \sim$ $\bar{n}^{0.75} R^{1.5}$. For the various $\mathrm{L}-\mathrm{H}$ transition mechanisms reviewed in Sec. 2.2, we have summarized the $\mathrm{H}$ mode power threshold scaling as $P_{t h r e s} \sim B^{\alpha} n^{\beta} R^{\gamma}$ in table 3 . 
Since the effect of finite parallel wavelength on the conducting-wall modes in the electromagnetic regime has been found to lowered growth rates and mixing-length diffusivities due to the reduction of perturbed parallel current by the electron skin depth. When a model is introduced for finite- $\beta$ and finite $k_{\|}$effects on the modes, we have shown that a power threshold for transition to the narrower root is obtained, suggesting a possible $\mathrm{L}-\mathrm{H}$ transition.

It is experimentally known that the outward current direction $U_{0}>0$ corresponds to an ion drift direction away from the X-point and the threshold is higher by a factor about two for an ion drift direction towards the X-point(normal drift direction). Cordey, Kerner, Pogutse and Nassigh found that for interchange mode with parallel

current $J_{\| 0}$ the linear instability growth rate varies as $\gamma \sim \frac{1}{2}\left(-\left(1-U_{0} \beta^{*}\right) \pm \sqrt{\left(1-U_{0} \beta^{*}\right)^{2}+\frac{4}{3} \beta^{* 2}}\right)$. By using mixing-length arguments, they found that for $U_{0}$ in the range of $-1<U_{0}<$ 1 , the diffusivity typically varies by a factor of 2 to 4 . We conduct similar biased simulations for a limiter tokamak, and the results are given in table 4 . By changing the variable $J_{\| 0} /$ nec $_{s}$ from +1 to -1 , the turbulence fluctuation levels and transport are significantly reduced. The heat diffusivity is reduced by a factor of 3 and the particle diffusion coefficient is reduced by a factor of 5 . This is consistent with experimental value and simple theoretical estimates. The reason is that the sheath impedance is larger at a target plate where the current flows into the plate. If the plate with larger sheath impedance is in the region of bad curvature, the decreased line-tying leads to destabilization and viceversa. In addition, the flow shear $\hat{U}_{0}(x)=\lambda \partial T_{\mathrm{eo}} / \partial x$ is smaller when current flows into the plate due to $\lambda$ is smaller. This further increases the instability.

\section{X-point Effects}

In Sec. 2 and Sec. 3 a simplified sheared magnetic field models are used. Tokamaks of current interest have divertors at the edge. The main complication in a diverted tokamak is the presence of the $\mathrm{X}$ point, which significantly changes the structure of the fluctuations due to magnetic shear or fanning effects. In an x-point divertor geometry, the local $q(r, \theta)$ profiles vary along the magnetic field line and there exists a steep parallel gradient of $q(x, \theta)$ near the x-point. There are two effects: (1) local magnetic shear becomes large near the $x_{\text {-point }}{ }_{i}(2)$ magnetic connection length becomes infinitely long when a flux tube passing near an $\mathrm{X}$ point. The following questions arise. Does the conventional ballooning mode representation break down? Does the huge variation of the local magnetic shear along the magnetic field line suppress instabilities such as resistive ballooning mode and conducting-wall mode? The separatrix is a unique flux surface which divides tokamak into two distinct physics regions: core and SOL. How do the turbulence in these two regions interact each other?

\subsection{Mode structure around a magnetic $\mathrm{X}$ point}

There are only a few theoretical studies of mode structures near $\mathrm{X}$ points. The mode structure of waves around a magnetic $\mathrm{X}$ point is analyzed by Mattor and Cohen 
using ray tracing techniques: They found two types of mode structure depending on the initial wavevector. In One, waves propagate around the X-point region, thus avoiding the large wave number increase from magnetic shear, and allowing them to correlate through such a region between mid-plane and divertor leg region. This effect is explicitly assumed with a break down of the eikonal approximation. In the other, the $\mathrm{X}$ point acts like potential hill to separate the main and diverto leg plasmas. The waves are reflected on either side. The remaining challenge is to continue the ray tracing analysis with boundary conditions to obtain the dispersion relation.

Within the eigonal approximation, the ballooning and sheath instabilities in the SOL of diverted plasmas is numerically analyzed in Ref.[] by Myra, D'Ippolito and Geoedbloed using a ballooning mode representation. The stability analysis includes the effects of curvature, resistivity, parallel variation of the $\mathbf{E} \times \mathbf{B}$ drift frequency, and sheath boundary conditions at the divertor plate. The numerical solutions indicates two modes: (i) the curvature-driven mode with growth rate enhanced by the sheaths (thus, a hybrid of curvature and conducting-wall modes); and (ii) the $\mathbf{E} \times \mathbf{B}$ axial shear mode driven by equilibrium variations in the region between the $\mathrm{X}$ point and the plate. The later mode is shown to be partly driven by the $\mathrm{X}$ point geometry. But the validaty of the eikonal representation is not addressed.

Ideal interchange instability near the separatrix with $x$ point geometry in the SOL has been studied using the reduced MHD model and applying a ballooning approximation to the perturbations by Kerner et al. 'Line-tying' boundary conditions are used at the target plates. It is shown that the SOL plasma just outside the separatrix can become unstable more easily than the plasma just inside the separatrix where the magnetic well is stabilizing. The criterion for the instability is $\hat{\beta}_{c r}=$ $q_{0}^{2} 4 \pi P_{0} / B_{0}^{2}>(\Delta x)_{x} / R_{0}$, where $(\Delta x)_{x}$ is the pressure gradient scale length at the separatrix at the $x$-point, while other quantities are measured at the mid-plane. For a typical divertor plasma, $\hat{\beta}_{c r}<<1$, thus the interchange instability is easy to develop near the $\mathrm{X}$ point. This is a mechanism to trigger the ELMs.

\subsection{D fluid turbulence simulations for realistic DIII-D divertor} geometry

We present results of 3D fluid simulations of tokamak edge turbulence with realistic parameters for a DIII-D divertor geometry. The basic set of equations is Eqs. (1)-(10) with the sheath boundary conditions Eq.(11) except that parallel ion velocity $v_{i \|}$ and the magnetic perturbation $\hat{A}_{\|}$have been set to zero. We incorporate the DIII-D configuration by reading magnetic field data from the UEDGE code. Quasiballooning coordinates are used with general geometry metric coefficients. The detailed treatment of geometry will be reported in future publication. The local safety factor $q(\psi, \theta)=h B_{T} / R B_{p}$ is plotted in Fig. 1 with conventional MHD safety factor defined as $q_{M H D}=\int d \theta\left(h B_{T} / R B_{p}\right) / \int d \theta$, where $h=\sqrt{R_{\theta}^{2}+Z_{\theta}^{2}}, \mathrm{R}$ is the major radius, $B_{T}$ toroidal field, $B_{p}$ the poloidal field. The striking variation of $q(\psi, \theta)$ across $\mathrm{X}$ point region is displayed. The normal and geodesic components of curvature are plotted in Fig. 2. The cross-field profiles of turbulence root-mean-square values of $\langle\phi\rangle,\langle n\rangle,,\left\langle T_{e}\right\rangle,\left\langle\chi_{e}\right\rangle$ and $\left\langle D_{i}\right\rangle$ are ploted in Fig.3. Instanenious plots 
of $\phi(x, y)$ and $\phi(y, z)$ are given in Fig. 4. We see that even with X point geometry, the turbulence fluctuation levels are still high. But the modes are located in the bulk plamsa and small beyond the X points. On the other hand, for an ideal $\left(\nu_{e i}=0\right.$ electromagnetic perturbation, linear eigonal ballooning analysis indicates that the mode can go beyond the $x$ point and connect to plate. The combination of finite electron collisionality and magnetic fanning tends to disconnect two regions. It remains to be seen how these electromagnetic effects manages themselves in a nonlinear and noneigonal simulation. Another interesting point is that the turbulence is stronger inside last closed flux surface than outside, which is opposite from the results in a limiter configuration.

\section{Summary}

We have presented recent developments in the area of the SOL turbulence theory and simulations, and its application to the $\mathrm{L}-\mathrm{H}$ transition. These developments includes the analysis and modeling electromagnetic turbulence, inclusion of complicated $X$ point divertor geometries, a unified description of various linear instabilities, the anomalous cross-field transport scaling, and power threshold scaling for $\mathrm{L}-\mathrm{H}$ transition.

The comparison of various theories with the experimental scaling in transport coefficients is not very encourage. There are several reasons. First, all the theoretical model scalings are based on mixing length estimates, and model specific. Thus more comprehensive nonlinear simulation runs are needed to determine functional dependence $F\left(\hat{\beta}, \rho_{s} / R_{0}, L_{\perp} / \rho_{s}, L_{\|} / r h o_{s}, \hat{\nu}, \Lambda\right)$ on the control parameters for the turbulence. Second, there is evidence that the transport in the SOL may not be diffusive. Large turbulence eddies on the order of the SOL width may convect from the separatrix out to the helo region. Hence development of transport scaling based on convection cells is desirable. Third, some portion of the SOL turbulence may come from propogation of turbulence generated in the core plasmas[Nathan].

Although we find that there is no strong connection between SOL and divertor plasma in our electrostatic nonlinear simulations. Linear results suggest that there maybe one correction when electromagetic effects are included. There is also the possible instabilities generated locally in the SOL divertor leg, either because of ionization and/or radiation drives or because of sheath generated local instabilities.[Ryutov and Cohen].

\section{Acknowledgments}

We thank Drs. T. D. Rognlien/LLNL, G. R. Porter/LLNL, Nathan Mattor/LLNL, Dmitri Ryutov/LLNL, Andris Dimits/LLNL, Jim Myra/Lodestar, R.A.Moyer/UCSD/GA, Bruce Scott /IPP-Garching, M Endler//IPP-Garching, D.R.McCarthy /Southeastern Louisiana Univ., J.Drake/Univ. Maryland, P.Guzdar/ Univ. Maryland, Hartmut Gerhauser/KFA-Juelich, H.A. Claassen /KFA-Juelich, Hubert Capes/EN-Cadarache, Xavier Garbet/EN-Cadarache, P. Ghendrih/N-Cadarache, Brian LaBombard/PSFCMIT, Spencer Pitcher/PSFC-MIT for enlightening physics discussions, and Alan Hindmarch/LLNL and Peter Brown/LLNL on using cvode PDE Solver software pack- 
age Performed for US DOE by LLNL under Contract W-7405-ENG-48.

\section{References}

[1] Moyer, R. A., Cuthbertson, J. W., Evans, T. E., Porter, G. D., Watkins, J. G., Journal of Nuclear Materials bf 241-243 (1997) 633-638.

[2] LaBombard, B., et al., Journal of Nuclear Materials bf 241-243 (1997) 149-166.

[3] Endler, M., Niedermeyer, H., Giannone, L., Holzhauer, E., Rudyj, A., Theimer, G., Tsois, N., and ASDEX TEAM Nuclear Fusion 35 (1995) 1307.

[4] Endler, M., Giannone, McCormick, K., Niedermeyer, H., Rudyj, A., Theimer, G., Tsois, N., Zoletnik, S., and ASDEX TEAM and W7-AS Team Physica Scripta 51 (1995) 1307.

[5] Endler, M., Giannone, McCormick, K., Niedermeyer, H., Rudyj, A., Theimer, G., Tsois, N., and ASDEX TEAM Journal of Nuclear Materials bf 220-222 (1995) 293-297.

[6] Hill, D. N., Journal of Nuclear Materials bf 241-243 (1997) 182-198.

[7] Cohen, R. H., Mattor, N., and Xu, X. Q., Contrib. Plasma Phys. 34 (1994) 232-246.

[8] Sanchez, E., Hidlgo, C., Pedrosa, M. A., Balbin, R., Garcia-Cortes, I., Estrada, T., and Milligen, B. Van, Transport, Chaos and Plasma Physics 2 (1997) 264.

[9] Sarazin, Y., Ghendrich, Ph., and Garbet, X., in European Physical Society Conference on Controlled Fusion and Plasma Physics, Vol. 1, pp.57, 1997.

[10] Nedospasov, A. V., in European Physical Society Conference on Controlled Fusion and Plasma Physics, Vol. 1, pp.344, 1997.

[1] Chankin, A. V., Plasma Phys. Control. Fusion 39 (1997) 1059-1082.

[2] Mattor, Nathan, Phys. Plasma 2 (1995) 4042.

[3] Rogers, B. N. and Drake, J. F., Physical Review Letters 79 (1997) 229

[4] Kerner, W., Nassigh, and Pogutse, O., Plasma Phys. Control. Fusion 39 (1997) 1101-1114.

[5] Myra, J. R., D'Ippolito, D. A. , and Geodbloed, Phys. Plasma 4 (1997) 1330.

[6] Kerner, W., Pogutse, O., and Linden, R Van der, Plasma Phys. Control. Fusion 39 (1997) 757-778.

[8] Cordey, J., Kerner, W., and Pogutse, O., Plasma Phys. Control. Fusion 37 (1995) 773-785.

[9] Cohen, R. H. and Xu, X. Q., Phys. Plasma 2 (1995) 3374.

[10] Cordey, J., Kerner, W., Pogutse, O., and Nassigh, A., Plasma Phys. Control. Fusion 37 (1996) 1095-1013.

[11] Pogutse, O., and Kerner, W., 21st EPS Conf. on Controlled Fusion and Plasma Physics (Montpellier, 1994) Vol. 18B, Part II, ed E Joffrini et al (Lausanne: Eurpoean Physical Society) p. 84.

[12] McCarthy, D. R., Booth, A. E., Hutchinson, E. J., Krasheninnikov, S. I., Phys. Plasma 4 (1997) 2567.

[13] McCarthy, D. R., Booth, Drake, J. F., and Guzdar, P. N., Phys. Plasma 4 (1997) 300 . 
[17] Cohen, R. H. and Ryutov, D. D., Phys. Plasma 4 (1997) 905. [19] Scott, B., Plasma Phys. Control. Fusion 39 (1997) 471-504.

[20] Scott, B., IPP 5/74, Max-Planck-Institute for Plasmaphysik April (1997).

[21] Xu, X. Q., Phys. of Fluids B5 (1993) 3641.

[22] Xu, X. Q., Cohen, R. H., Plasma Phys. Control. Fusion 35 (1993) 1071.

[23] Berk H L, Cohen R H, Ryutov D D, Tsidulko Yu, and Xu X Q 1993 Nuclear Fusion 33 263-282

[24] Tsidulko Yu, Berk H L, and Cohen R H, Phys. of Plasmas 1 (1994) 1199.

\section{Figures}

Fig. 1: The model equilibrium profiles for electron temperature, density and parallel ionvelocity.

Fig. 2: The rms values of fluctuating potential $<e \tilde{\phi}(x, \chi, \zeta) / T_{e 0}(x, \zeta)>_{r m s}$, electron temperatue $\left\langle\tilde{T}_{e}(x, \chi, \zeta) / T_{e 0}(x, \zeta)\right\rangle_{r m s}$, density $\left\langle\tilde{n}_{e}(x, \chi, \zeta) / N_{e 0}(x, \zeta)\right\rangle_{r m s}$, and parallel ion velocity $\left\langle V_{i}(x, \chi, \zeta) / V_{i 0}(x, \zeta)\right\rangle_{r m s}$.

Fig. 3: The turbulent heat, particle and momentum diffusivity. 
Table 1: Characteristic Scale length and Time in SOL

\begin{tabular}{|l|l|l|l|l|l|}
\hline Physics & Ion gyro-physics & Collisonless skin & Collisional skin & Velocity shear & Drift Turbulence \\
\hline \hline Time & $\omega_{c i} \sim 10^{8}$ & $\omega_{p e} \sim 10^{11}$ & $\omega_{*} \sim 5 \times 10^{5}$ & $V_{E 0}^{\prime} \sim 1 \times 10^{5}$ & $\gamma_{\max } \sim 1 \times 10^{5}$ \\
\hline Length & $\rho_{s} \sim .5 \mathrm{~mm}$ & $\delta=\frac{c}{\omega_{p e}} \sim 1 \mathrm{~mm}$ & $\delta_{c}=\frac{D_{\text {eki }}}{\omega_{c}} \sim 1 \mathrm{~mm}$ & $L_{T e} \sim 5-10 \mathrm{~mm}$ & $k_{\perp} \rho_{s} \sim 0.1$ \\
\hline
\end{tabular}


Table 2: Comparison of Code Results for finite beta

\begin{tabular}{|l|l|l|l|l|l|l|l|l|}
\hline Effects & $\left\langle\widetilde{B} / B_{0}\right\rangle$ & $\langle\phi\rangle$ & $\left\langle N_{i}\right\rangle$ & $\left\langle T_{e}\right\rangle$ & $\left\langle V_{i}\right\rangle$ & $\left\langle j_{\|}\right\rangle$ & $\left\langle\chi_{e}\right\rangle$ & $\left\langle D_{i}\right\rangle$ \\
\hline \hline ES & 0.0 & 0.173 & 0.116 & 0.052 & 0.00469 & 4.3 & 3.04 & 5.32 \\
\hline EM & $3.4 \times 10^{-4}$ & 0.258 & 0.11 & 0.157 & 0.0316 & 3.92 & 5.6 & 4.4 \\
\hline DIII-D & & $0.15-0.3$ & $0.3-0.4$ & & & & & $1.3-0.52$ \\
\hline
\end{tabular}


Table 3: Comparison of H-mode Power Threshold Scaling. note: $B$ is the toroidal field in tesla, $n$ the average density, $\mathbf{R}$ the major radius in meter. The number is exponent of the corresponding variable in the formula: $P \sim n^{\alpha} B^{\beta} R^{\gamma}$. For the difference between SNL and DNL, see Ref.11

Experimental Scaling

\begin{tabular}{|l|l|l|l|l|}
\hline Models & ITER & JET & DIII-D(SNL) & DIII-D(DNL) \\
\hline$\alpha(B)$ & 1 & 1 & 1 & 0 \\
\hline$\beta(n)$ & 1 & $0.5-0.75$ & 1 & 0 \\
\hline$\gamma(R)$ & 2 & 1.5 & & 1 \\
\hline
\end{tabular}

Theoretical Scaling

\begin{tabular}{|l|l|l|l|l|}
\hline Models & CX & CKP(SNL) & CKP(DNL) & Chankin \\
\hline \hline$\alpha(B)$ & 0.74 & -1 & $3 / 5$ & 1 \\
\hline$\beta(n)$ & 0.75 & 3 & $3 / 5$ & 1 \\
\hline$\gamma(R)$ & 1 & 4 & $16 / 5$ & 1.75 \\
\hline
\end{tabular}

$\mathrm{CX}-$ Cohen and $\mathrm{Xu}$

CKP(SNL) - Cordey, Kerner and Pogutse; Single Null Divertor

CKP(DNL) - Cordey, Kerner and Pogutse; Double Null Divertor 
Table 4: Comparison of Local Transport Scaling for L-mode in experiments. note: The number is exponent of the corresponding variable in the formula: $\chi_{\perp} \sim n^{\alpha} B^{\beta} L^{\gamma} T_{e}^{\sigma}$. For the difference between SNL and DNL, see Ref.11

\begin{tabular}{|c|c|c|c|c|c|}
\hline \multicolumn{6}{|c|}{ Experimental Scaling } \\
\hline Models & DIII-D & C-MOD & ASDEX(1) & ASDEX(2) & JET \\
\hline$\alpha(B)$ & & $\overline{0}$ & $\overline{0}$ & & \\
\hline $\overrightarrow{\beta(n)}$ & -0.78 & -0.6 & & & -1. \\
\hline$\gamma(L)$ & -0.75 & -0.7 & & & \\
\hline$\sigma\left(T_{e}\right)$ & $2.25(-0.25)$ & -0.6 & 1 & -1 & -1 . \\
\hline
\end{tabular}

Theoretical Scaling

\begin{tabular}{|l|l|l|l|l|l|}
\hline Models & Bohm & BCRTX & Chankin & Endler(1) & Endler(2) \\
\hline$\alpha(B)$ & -1 & $-6 / 5$ & $-4 / 3$ & $-4 / 3$ & $-4 / 3$ \\
\hline$\beta(n)$ & 0 & 0 & 0 & 0 & $2 / 3$ \\
\hline$\gamma(L)$ & 0 & $-1 / 5$ & $-1 / 3$ & $1 / 3$ & 1 \\
\hline$\sigma\left(T_{e}\right)$ & 1 & $11 / 10$ & $7 / 6$ & $7 / 6$ & $-1 / 6$ \\
\hline
\end{tabular}

BCRTX-Berk, Cohen Ryutov, Tsidulko, and Xu

Endler(1) - Collisionless

Endler (2) - Collisional 
Table 5: Comparison of Code Results for Biasing, $U_{0}=J_{\| 0} / n e$

\begin{tabular}{|l|l|l|l|l|l|l|l|l|}
\hline$U_{0} / c_{s}$ & $\left\langle\vec{B} / B_{0}\right\rangle$ & $\langle\phi\rangle$ & $\left\langle N_{i}\right\rangle$ & $\left\langle T_{e}\right\rangle$ & $\left\langle V_{i}\right\rangle$ & $\left\langle j_{11}\right\rangle$ & $\left\langle\chi_{e}\right\rangle$ & $\left\langle D_{i}\right\rangle$ \\
\hline \hline 1 & & 0.0965 & 0.141 & 0.0318 & 0.0093 & 0.708 & 0.00016 & 0.000371 \\
\hline 0 & $2 \times 10^{-5}$ & 0.033 & 0.05 & 0.03 & 0.0033 & 0.09 & 0.00013 & $7.6 \times 10^{-5}$ \\
\hline-1 & & 0.0282 & 0.066 & 0.0169 & 0.0033 & 0.283 & $5 \times 10^{-5}$ & $6.06 \times 10^{-5}$ \\
\hline
\end{tabular}




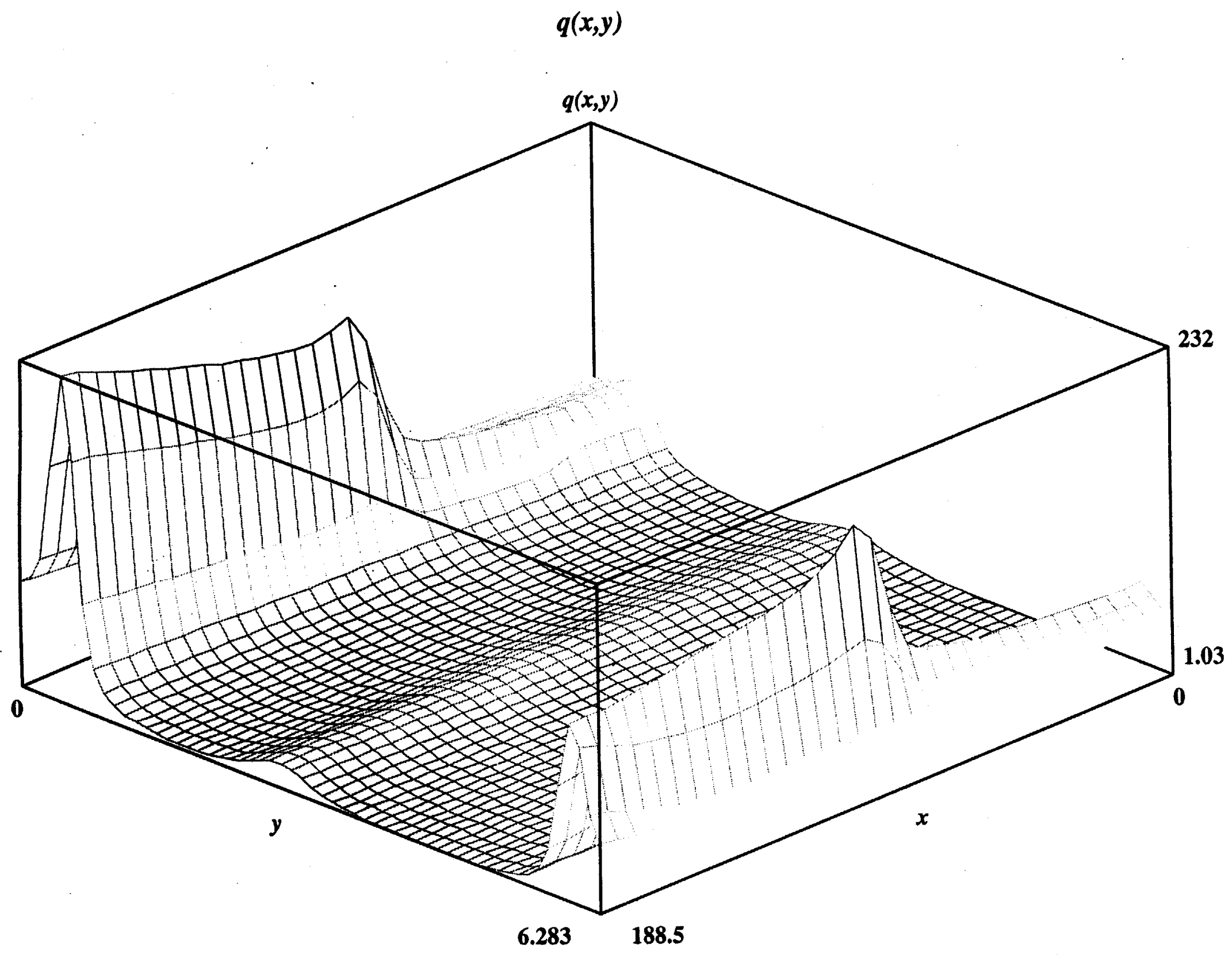

Fig. 1 


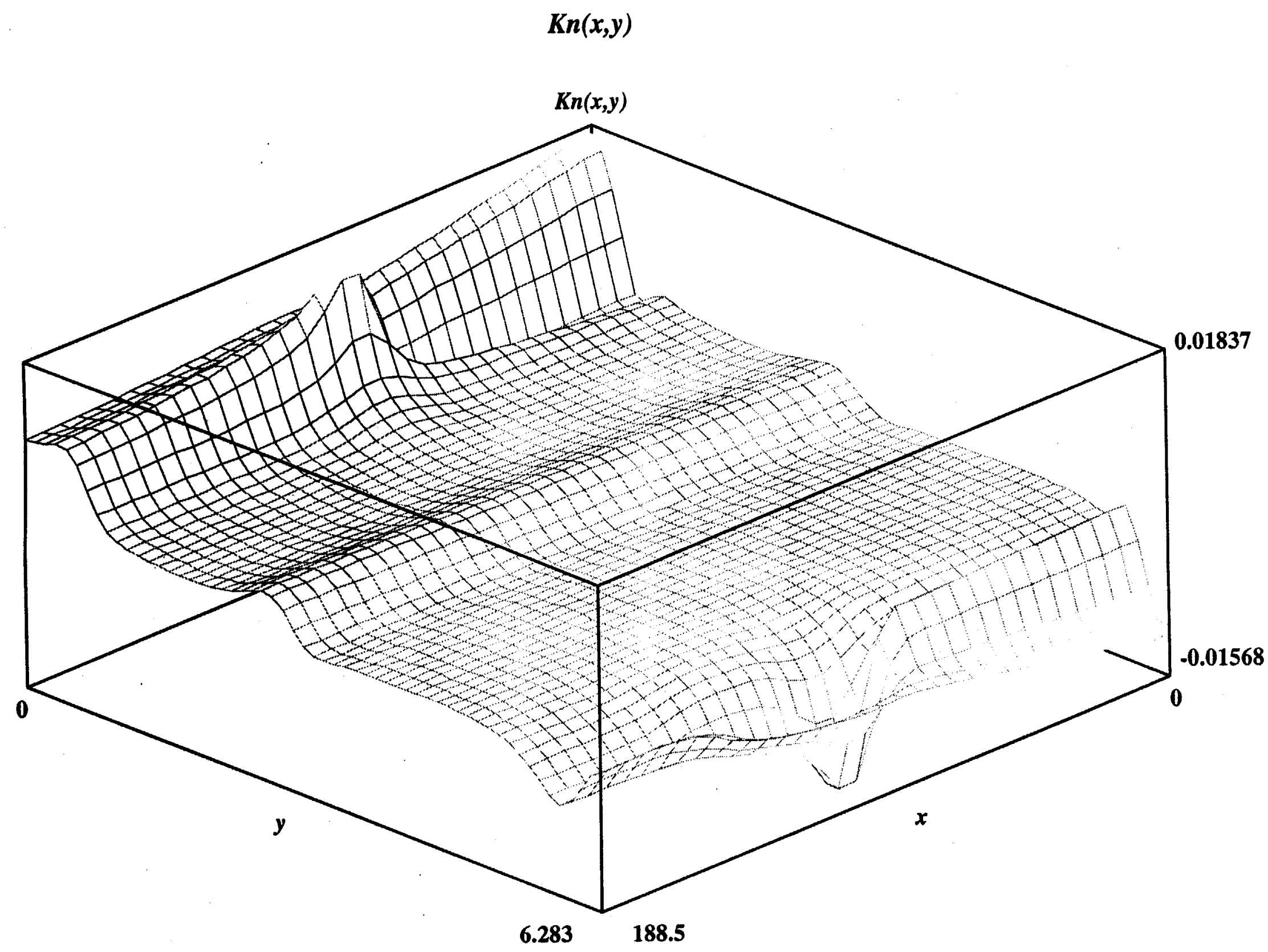

$F i g z a$ 


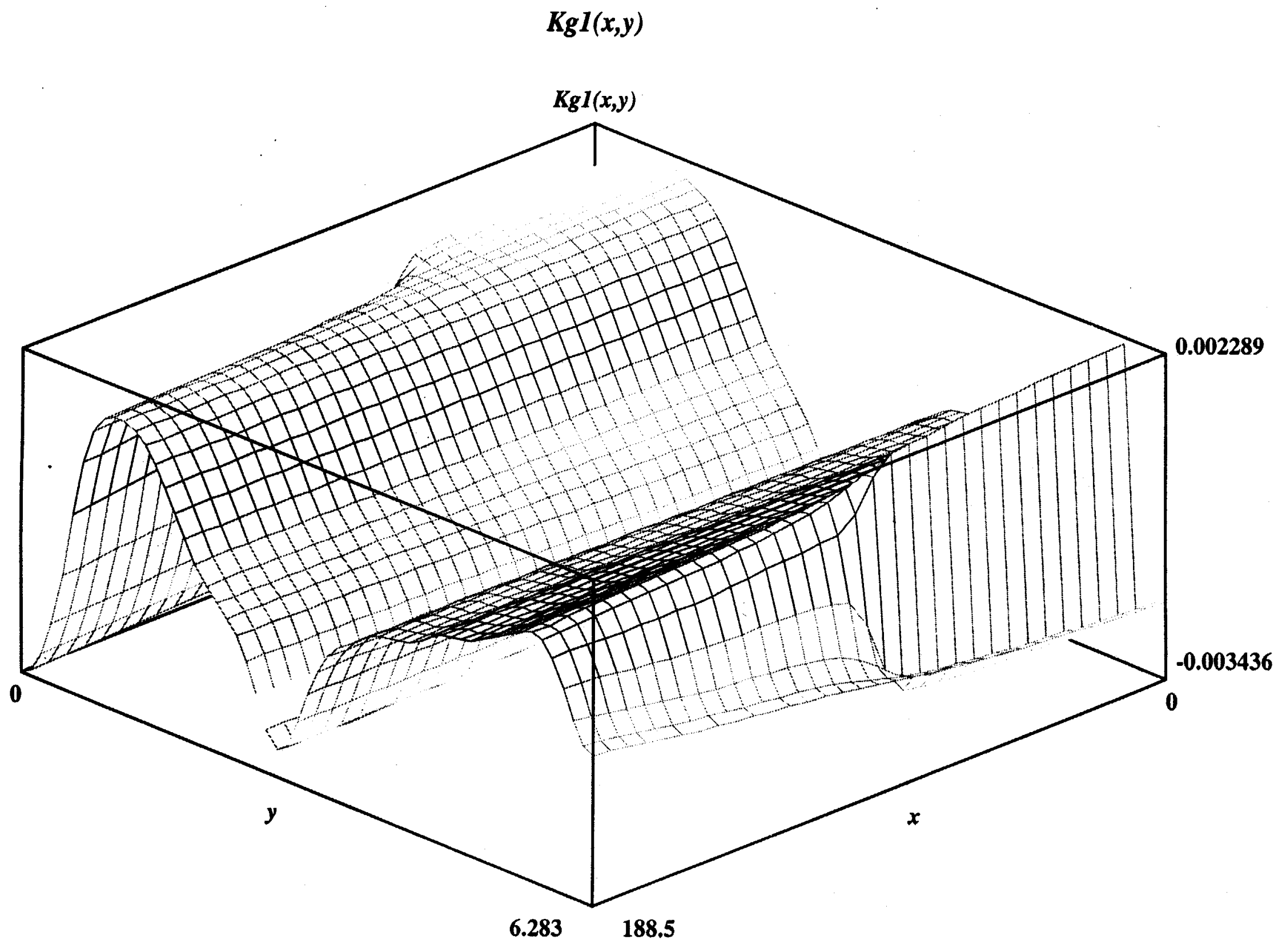

$F_{i g}=b$. 


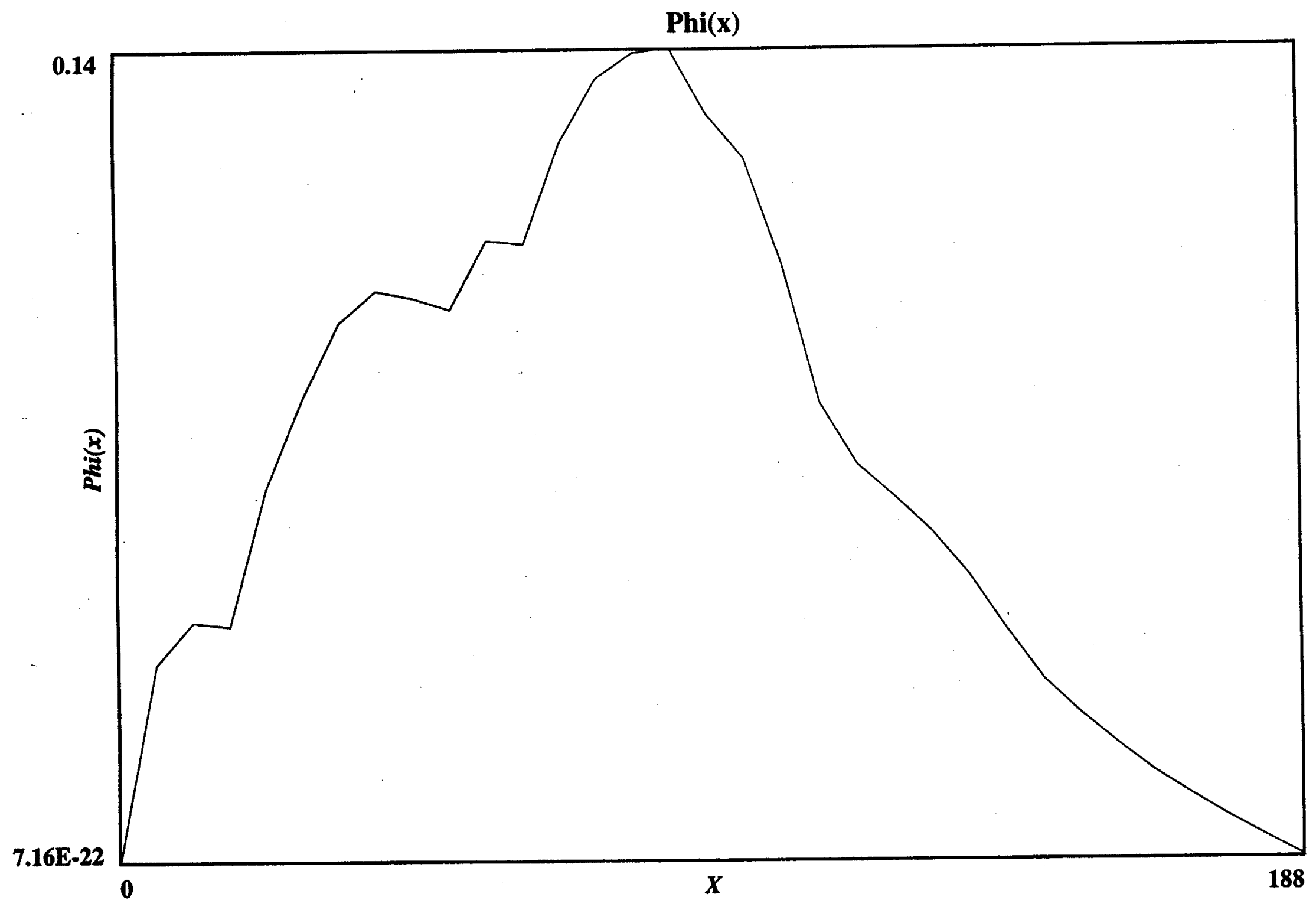

Fig $3 a$ 


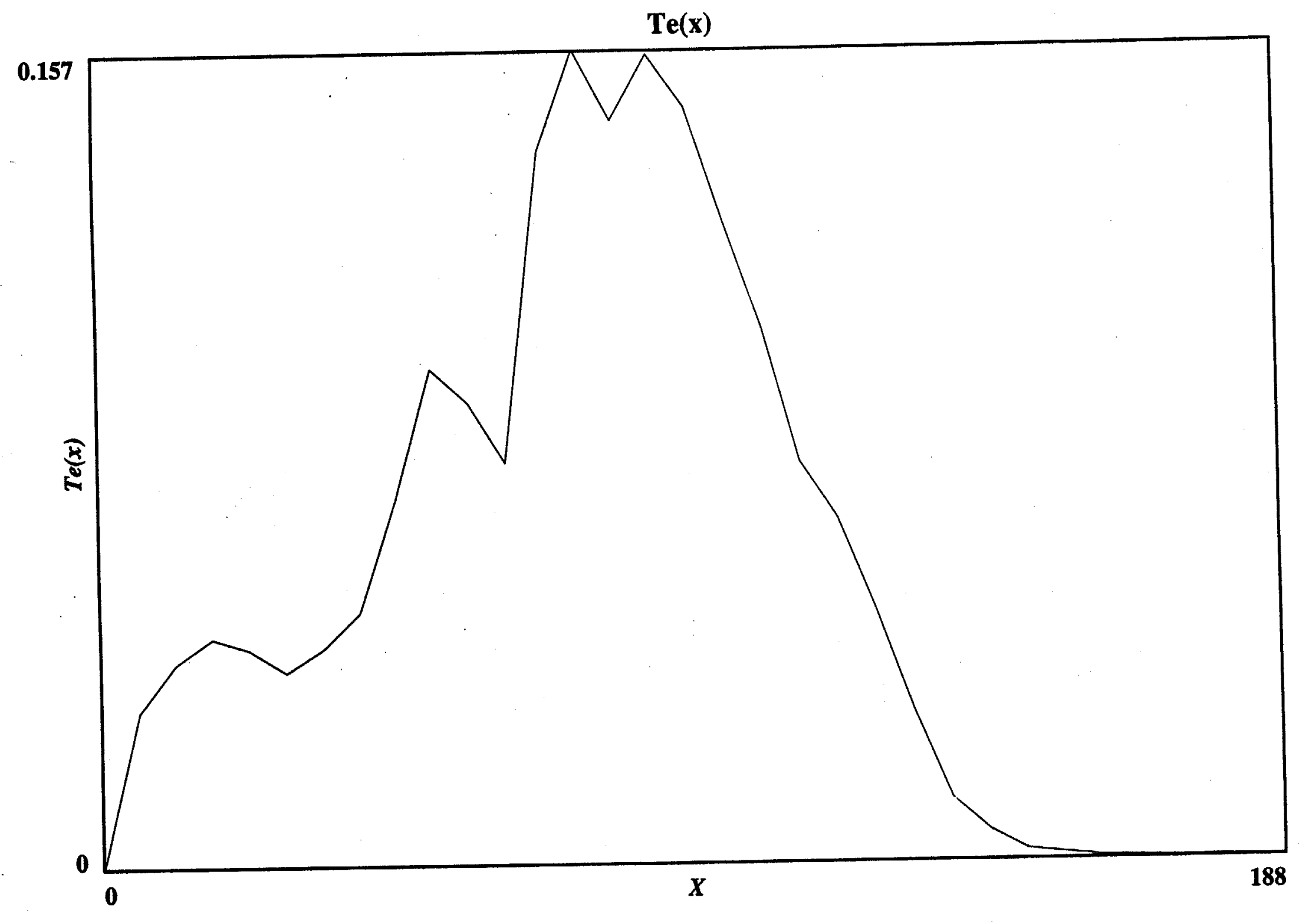

Fig36 


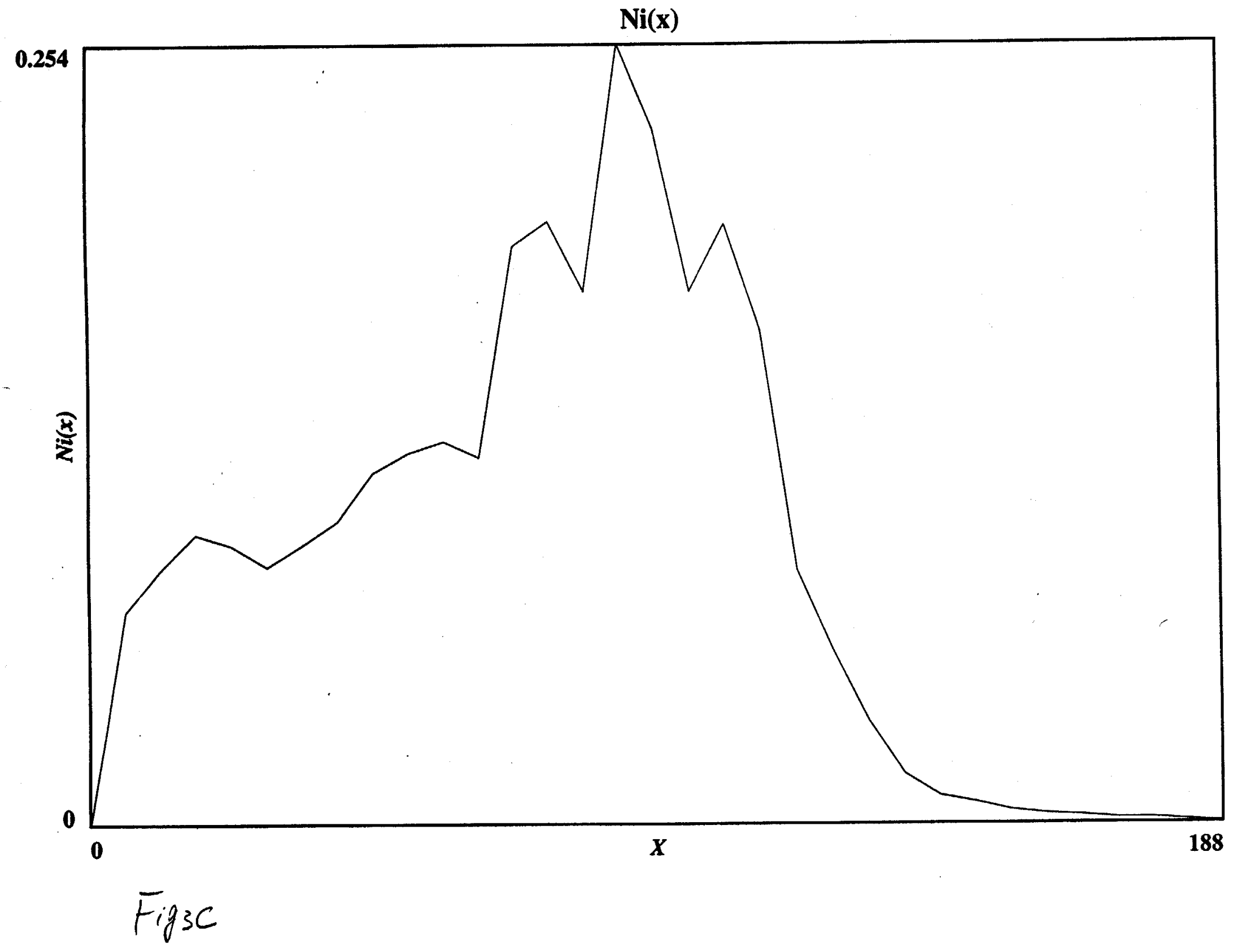




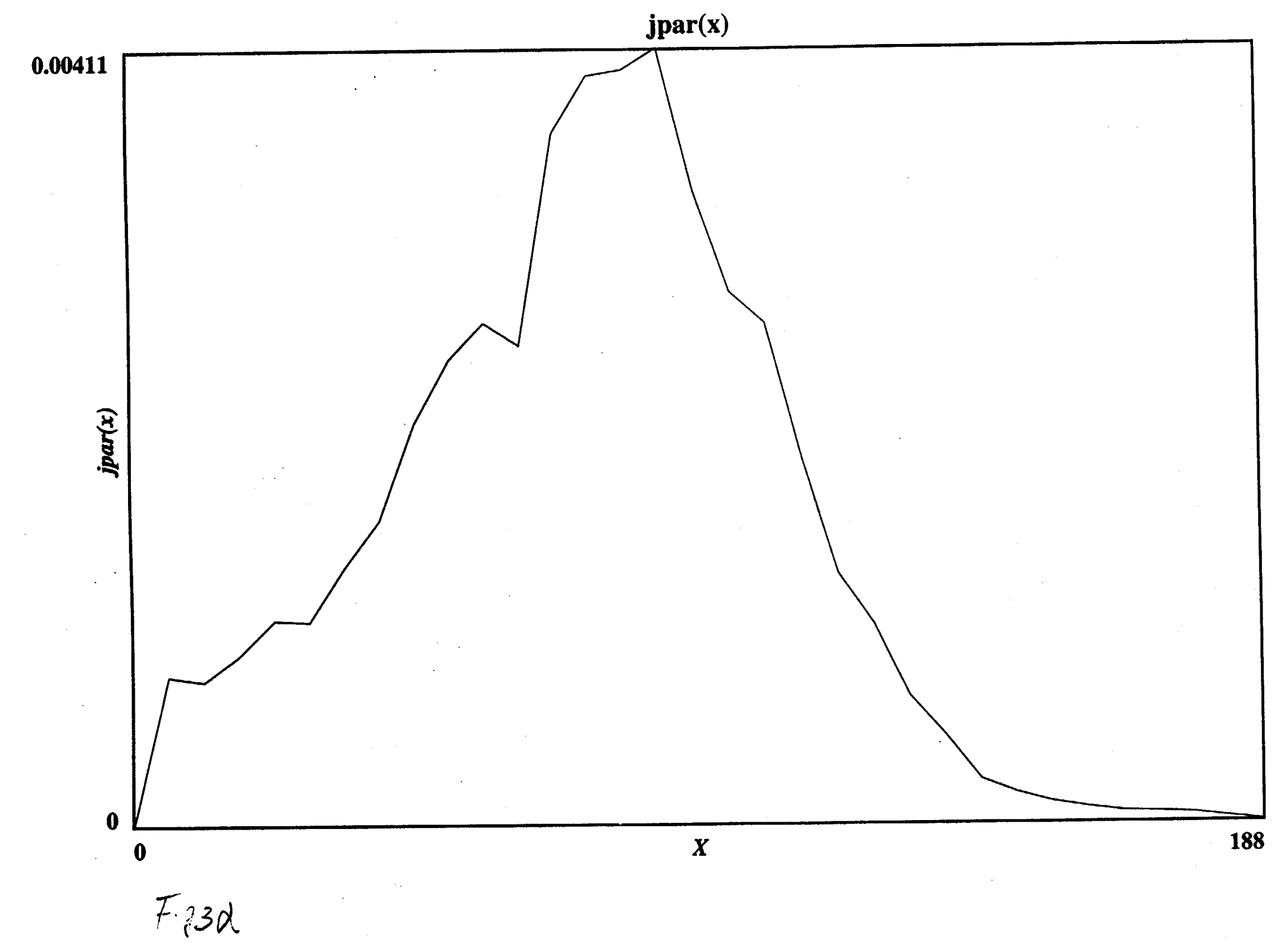


$\operatorname{Texy}(x, y)$

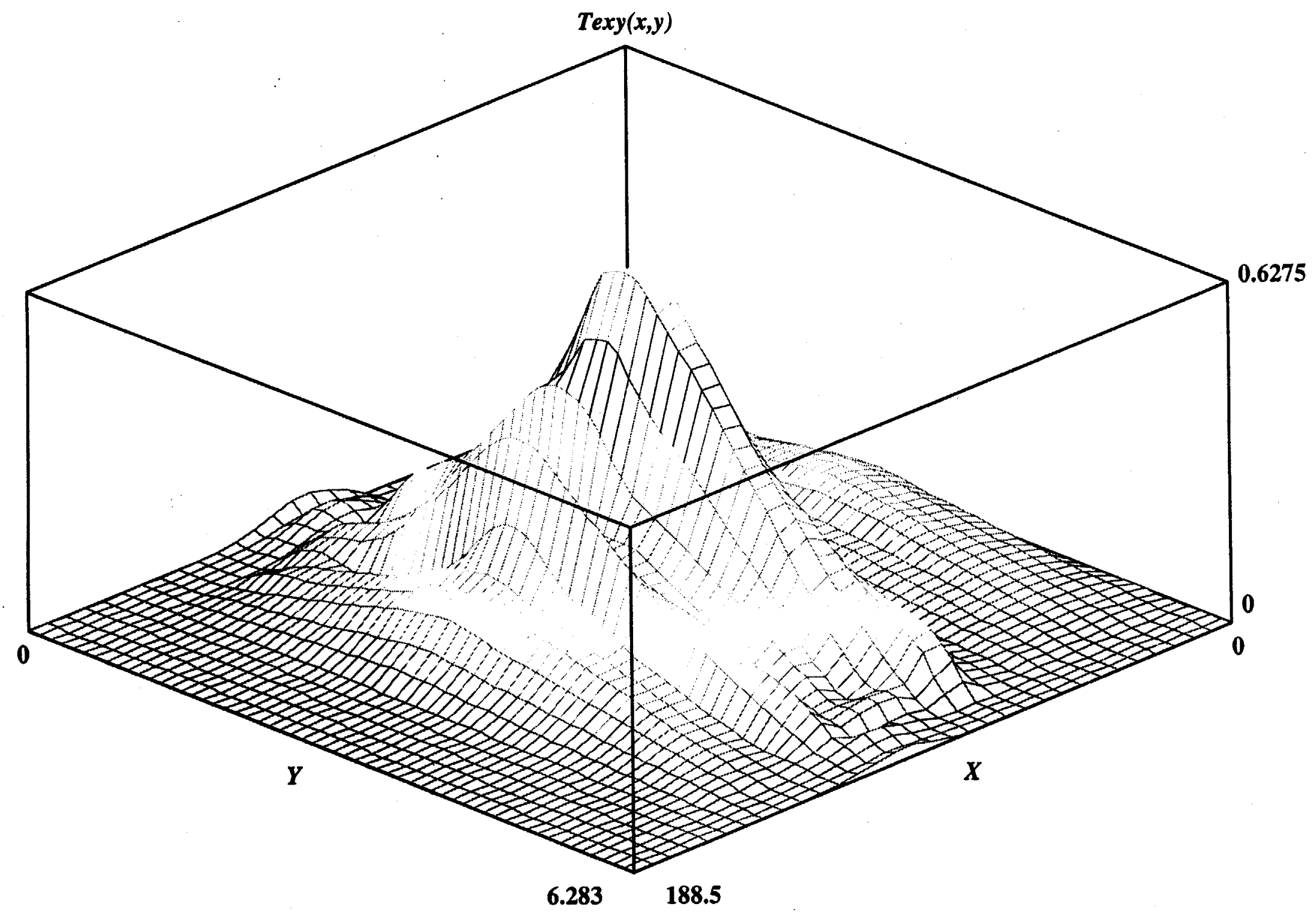

Fig $4 \lambda$ 


\section{$T e y z(y, z)$}

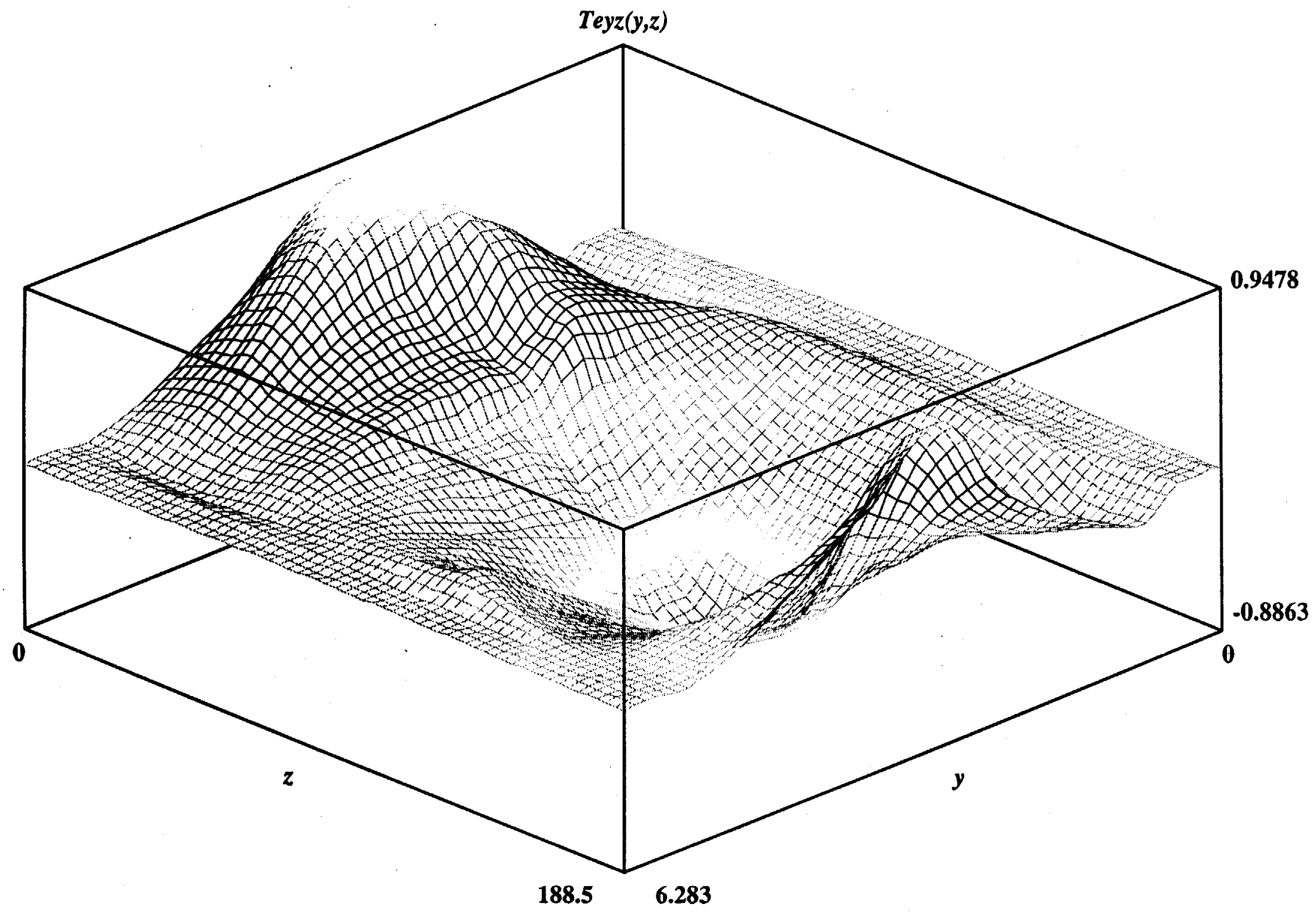

Fiofb 


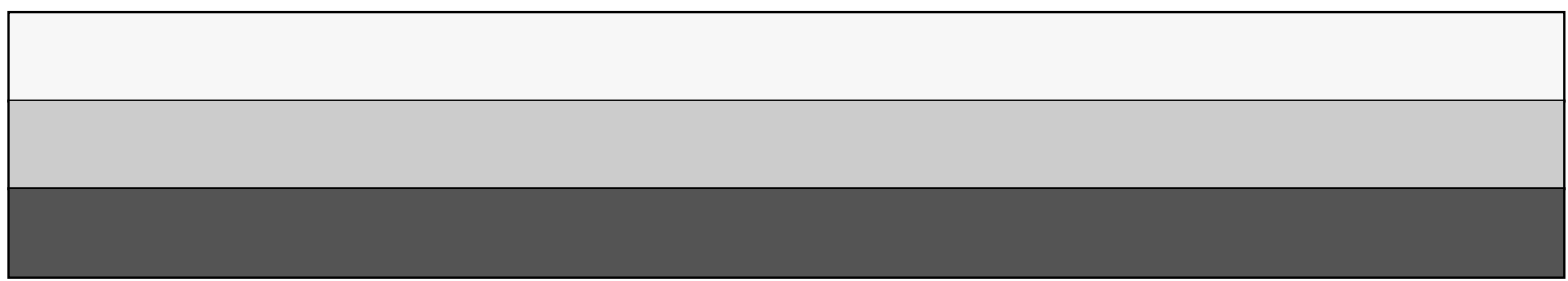

